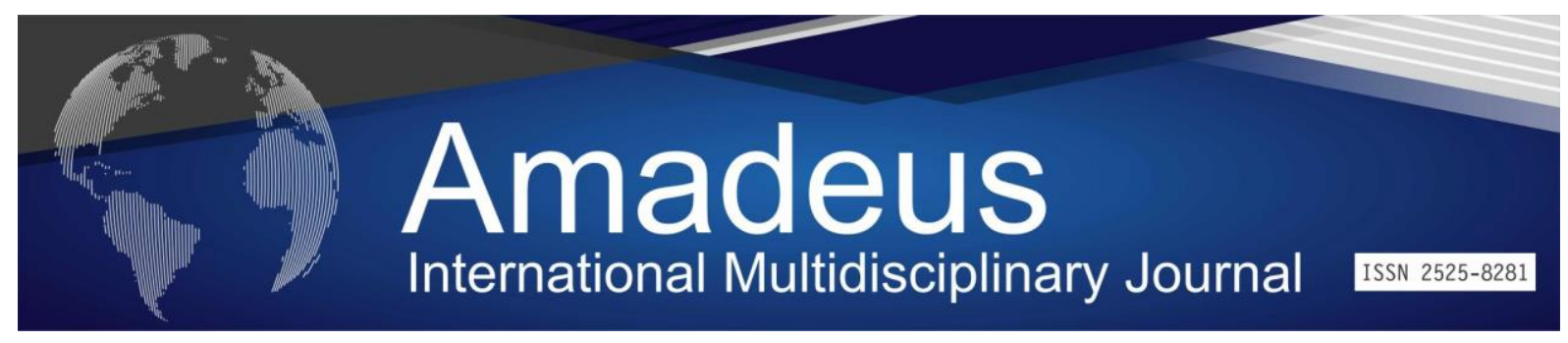

DOI: 10.14295/aimj.v5i9.117

\title{
Cognitive-Behavioral Therapy: An Ally of the Unified Health System
}

Rafael Zaneripe de Souza Nunes ${ }^{l}$, Lisiane Tuon ${ }^{2}$ Rosimeri Vieira da Cruz de Souza ${ }^{3}$, Karin Martins Gomes ${ }^{4}$
Abstract: The Unified Health System goes through several challenges, including the crisis related to public financing. The consequences generated by this crisis also affect the mental health network, which proves to be one of the most fragile networks in the public health system. As a result of the barriers encountered, this article seeks to analyze in the scientific literature how Cognitive-Behavioral Therapy can contribute to the better use of public spending through its objective and brief techniques. The study is characterized by a qualitative approach carried out through a nonsystematic literature review in the data sources SCIELO, BVS, PUBMED® and Google Scholar. Although the number of articles found in the national literature is limited, studies conducted outside the country demonstrate the effectiveness of CBT in public health systems, especially regarding the remission of users' symptoms and the cost-benefit of public spending.

Keywords: Cognitive Behavioral Therapy; Public Health; Unified Health System; Government Financing.

\footnotetext{
${ }^{1}$ Psicólogo Especialista e Mestrando em Saúde Coletiva pela Universidade do Extremo Sul Catarinense - UNESC. rafaelzaneripe.psico@gmail.com;

${ }^{2}$ É Doutora em Medicina e Ciências da Saúde; Professora do Programa de Pós-graduação - Mestrado em Saúde Coletiva da UNESC; Coordenadora e Tutora da Residência Multiprofissional da UNESC; e Coordenadora do Núcleo de Saúde Coletiva da UNESC. Já foi Coordenadora do Programa de Pós-graduação - Mestrado em Saúde Coletiva da UNESC; Superintendente de Serviços Especializados e Regulação da Secretaria de Estado da Saúde de Santa Catarina, onde atuou na coordenação e implantação da Rede de Urgência e Emergência, da Rede da Pessoa com Deficiência e das Centrais de Regulação por todo o Estado de Santa Catarina. Fez a implantação e coordenou do Centro Especializado em Recuperação CER/UNESC. Tem por linhas de pesquisas Gestão em Saúde; Epidemiologia; e Pessoa com Deficiência, com parceria junto a Federação das APAES do Estado de Santa Catarina. Possui dezenas de artigos publicados, livros e capítulos de livros, além de projetos de pesquisa em curso. 1tb@unesc.net;

${ }^{3}$ Possui graduação em Psicologia pela Universidade do Extremo Sul Catarinense. Pós-graduação em Psicopedagogia Escolar, Especialização em Teoria Cognitiva Comportamental, Mestrado em Administração pela UNISUL - Florianópolis. Experiência na área de Psicologia, com ênfase em Psicologia do Trabalho e Organizacional, Formação em Coaching e Neurocoaching para Lideranças, Capacitação pela FGV em RH Estratégico. Faz parte do corpo docente do Curso de Psicologia da Universidade do Extremo Sul Catarinense e Pós-graduação. rosimerivieira@unesc.net;

${ }^{4}$ Possui graduação em Psicologia pela Universidade do Vale do Itajaí (2003), mestrado em Ciências da Saúde pela Universidade do Extremo Sul Catarinense - UNESC(2006) e doutorado em Ciências da Saúde pela Universidade do extremo sul catarinense - UNESC (2009). Possui espacialização em neuropsicologia e formação em Terapia Cognitiva. É tutora da residência multiprofissional - UNESC e esta como coordenadora do Curso de Psicologia - UNESC, é docente do curso de psicologia da UNESC. karin@unesc.net.
} 


\title{
Terapia Cognitivo-Comportamental: Uma Aliada do Sistema Único de Saúde
}

\begin{abstract}
Resumo: O Sistema Único de Saúde passa por diversos desfios, entre eles a crise relacionada ao financiamento público. As consequencias geradas por essa crise também afetam a rede de saúde mental, que demonstra ser uma das mais frágeis do sistema púbico de saúde. Visto as barreiras encontradas, o presente artigo busca análisar na literatura científica como a Terapia Cognitivo-Comportamental pode contribuir no melhor aproveitamento dos gastos públicos através de suas técnicas objetivas e breves. O estudo é caracterizado por uma abordagem qualitativa realizado através de uma revisão não-sistemática de literatura nas fontes de dados SCIELO,BVS, PUBMED® e Google Acadêmico. Embora o número de artigos encontrados na literatura nacional seja limitado, estudos realizados fora do país demonstram a eficácia da TCC nos sistemas públicos de saúde, principalmente no que refere à remissão dos sintomas dos usuários e no custo-benefício de gastos públicos.
\end{abstract}

Palavras-chave: Terapia Cognitivo-Comportamental; Saúde Pública; Sistema Único de Saúde; Financiamento Governamento.

\section{Introdução}

O Sistema Único de Saúde criado a partir da Constituição Federal (Brasil, 1988) e regulamentado pelas leis 8080 (Brasil, 1990a) e 8142 (Brasil, 1990b) é um sitema universal e gratuito que atende a toda população circunscrita no território brasileiro. Arruda (2015), discorre acerca das Redes de Atenção (RAS) no SUS, e afirma que elas se configuram como um sistema de múltiplas conexões e inter-relações, contenectando pontos distintos de atenção, profissionais e usuários.

Dentre as diversas redes de atenção no SUS, destacamos a RAPS ou Rede de Atenção Psicossocial (Brasil, 2011), que seria o componente do sistema responsável pelo oferecimento de ações e serviços voltados a pessoas com transtornos mentais, em sofrimento psiquico e com necessidades decorrentes do uso de álcool e outras drogas. Os componentes dessa rede incluem os pontos de atenção básica; atenção psicossocial especializada; atenção de urgência e emergência; atenção hospitalar e outros.

Devido às circustâncias economicas atuais, o SUS vem sofrendo com uma baixa alocação de recursos, o que levou a uma crítica negativa de vários autores com relação à EC 
95, que limitava os gastos públicos em saúde (Paim, 2018; Santos, 2018a; Mendes; Carnut \& Guerra, 2018; Funcia, 2019; Menezes, Moretti \& Reis, 2020). Obviamente os aspectos financeiros refletem na estruturação e efetivação das políticas públicas e nos serviços oferecidos aos usuários (Santos, 2018b; Melo, Almeida, Lima \& Giovanella, 2020). Entretanto, levanta-se a questão: em um contexto de estabilidade financeira no país, agravado pela pandemia do novo coronavírus, de que forma podem-se melhorar os serviços com os recursos disponíveis?

Sendo assim, esse artigo objetiva trazer uma reflexão sobre possíveis contribuições da Terapia Cognitivo-Comportamental como uma abordagem baseada em evidências que pode contribuir no direcionamento e investimento eficaz dos recursos públicos em saúde mental no Sistema Único de Saúde.

\section{Método}

O presente estudo caracteriza-se como um ensaio teórico descritivo qualitivo, realizado através de uma revisão não-sistemática de literatura. O objetivo principal é ver as possíveis benefícos do uso da Terapia Cognitivo-Comportamental nos sitemas públicos de saúde, especialmente no Sistema Único de Saúde brasileiro. Esse tipo de estudo é apropriado para descrever e discutir o desenvolvimento de determinado assunto, seja pelo aspecto téorico ou contextual (Rother, 2007).

A coleta de dados foi realizada através das plataformas de pesquisa Scientific Eletronic Library Online (SCIELO), Biblioteca Virtual em Saúde (BVS), PUBMED® e Google Acadêmico, com os descritores "Terapia Cognitivo-Comportamental" e "Saúde Pública"; "Terapia Cognitivo-Comportamental" e "SUS"; "Cognitive Behavioral Therapy" e "Public Health"; "Cognitive Behavioral Therapy" e "Health System"; "Cognitive Behavioral Therapy" e "Unified Health System". Utilizando na busca o booleano "and". 
Figura 1 - Base de dados utilizada para a composição da seção temática.

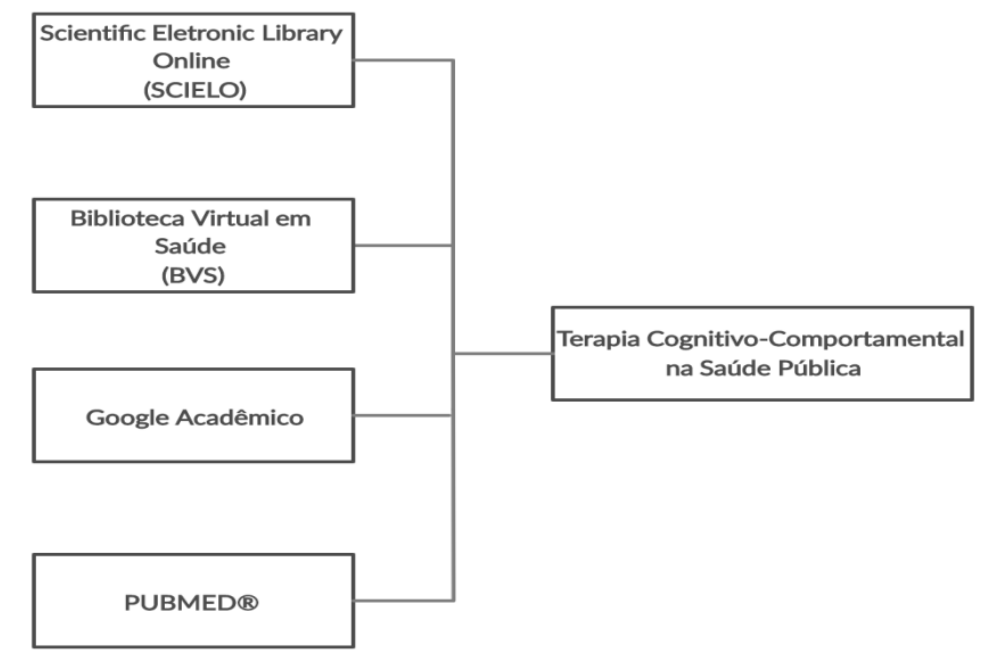

Fonte: Autores do estudo (2020)

A análise dos dados ocorreu através das etapas de pré-análise, exploração do material e tratamento dos resultados sugerido por Bardin (2009). Após a seleção e organização dos dados, foi realizada a categorização em uma unidade de análise afim da interpretação dos dados. Essa seção foi nomeada como: "Terapia Cognitivo-Comportamental na Saúde Pública". Optou-se por elaborar uma seção temática independente a fim de subsidiar os poucos dados encontrados na seção supracitada, no qual nomeamos de "Terapia CognitivoComportamental como uma abordagem baseada em evidências".

\section{Terapia Cognitivo-Comportamental como uma Abordagem Baseada em Evidências}

A Terapia Cognitivo-Comportalmental, conhecida também como "TCC", foi concebida por Aaron Beck no início da década de 60. Na epóca, Dr. Beck procurava comprovar a eficácia do modelo psicodinâmico no tratamento a depressão, e através da prática clínica identificou uma série de pensamentos negativos que estavam ligados às emoções e comportamentos desadaptativos dos pacientes, inciando assim a formulação da abordagem cognitivo comportamental (Beck, 2013).

Conforme Wright, Brownm Thase \& Basco (2019) a TCC é uma abordagem de senso comum e se baseia em dois principios centrais: as cognições influênciam nossas emoções e 48 
comportamentos (conforme citado anteriormente) e o modo como agimos ou nos comportamos afeta nossos padrões de pensamentos e emoções. Isso vai de encontro com os aspectos descritos por Dobson \& Dozois (2006), que ainda acrescenta que a cognição pode ser monitorada e altera, e que a mudança comportamental desejada pode ser efetivada através da mudança cognitiva. Aliado a isso, devido a sua forte ligação com a pesquisa científica, a TCC domina as diretrizes internacionais para tratamentos psicossociais, sendo considerado o tratamento de primeira linha para muitos distúrbios e respaldada pela American Psychological Association (David, Cristea \& Hofmann, 2018).

Essa abordagem surgiu no cenário americano marcado por uma forte tradição que enfatiza a necessidade de fundamentação científica e o desenvolvimento de práticas clínicas com eficácias comprovadas. Além disso, se tratanto da psicologia clínica contemporânea, a avaliação da eficácia das psicoterapias é imprensciendivel antes de disponibilizar o tratamento ao público (Mariano, 2019). Outro fator que apoia sua base científica é a constante evolução nas estruturas de sua prática, que seguem o fluxo progressivo de novos dados obtidos, diferente de outras abordagens na psicologia (David, Cristes \& Hofmann, 2018) Isso pode ser visto inclusive por meio dos resultados obtidos através da TCC em sintomas ansiosos, que acabou influenciando a criação de um protocolo padronizado por Pombo \& Ferro (2018) para pacientes que apresentassem ataques de pânico e crises de ansiedade.

A TCC dispõe de diversas evidências científicas, sendo uma interveção poderosa para problemas de saúde mental (Dobson \& Dobson, 2008). A abordagem cognitiva já foi amplamente testada desde a publicação de seus primeiros estudos, e hoje demonstra sua eficácia no tratamento de diversas patologias e problemas psicológicos, tais como: Depressão; Transtorno de Ansiedade Generalizada; Transtorno Obssesivo-Compulsivo; Transtornos de Personalidade; Transtorno Bipolar; Problemas Conjugais; Problemas Familiares; Luto complicado e várias outras situações (Beck, 2013). Também é importante frizar que o empirismo colaborativo, as técnicas de estruturação, a ênfase psicoeducacional, os métodos pragmáticos e a tarefas de casa (características elementares da TCC) aliadas a farmacoterapia podem ser extremamente utéis para sessões breves e de alto rendimento (Wright, Sudak, Turkington \& Thase, 2012).

Conforme Mariano (2019), o movimento do cientista clínico sofre algumas críticas em virtude da sua comcepção não dar um espaço claro para a utilização do saber hermenêutico no contexto clínico, sendo seu foco dirigido objetivamento a diminuição ou alívio de determinada sintomatologia. Isso poderia dar ares de um estrito cientificismo por parte da 
TCC, mas de acordo Martins et al. (2018), Aaron Beck sempre considerou a empatia como uma variante importante na relação terapeutica dentro da abordagem cognitiva, onde tal habilidade poderia ser desenvolvida e aperfeiçoada pelos terapeutas.

A TCC acaba sendo o padrão ouro no campo da psicoterapia pelo sua rigorosa base empírica nos campos da ciência e pesquisa moderna, e não por razões políticas como alguns profissionais possam sugerir (David, Cristes \& Hofmann, 2018). As diversas possibilidades de aplicação da abordagem podem inclusive servir como modelo clínico de avaliação e intervenção em problemas neurológicos como demonstrado por Charchat-Fichman, Fernandes \& Ladeira-Fernandez (2012).

Jokić-Begić afirma que as intervenções da TCC alteram o funcionamento do cérebro associados à resolução de problemas, processamento auto-referencial e relacional, além de afetar a regulação cerebral. O autor também afirma que numa análise das imagens do cérebro pré e pós-tratamento, foi possível observar mudanças nas estruturas corticais e subcorticais, e que a terapia influencia na regulação cerebral de cima para baixo, ou seja, as mudanças obtidas podem ser permanentes e afetarem diferentes áreas da vida do paciente.

Referente ao Brasil, a popularidade da terapia cognitiva decorre também da sua eficácia na prática clínica, onde seu desenvolvimento no país ocorreu no final da década de 80, numa época que os recursos para obtenção de acesso à literatura científica internacional eram mais difíceis (Rangé, Falcone \& Sardinha, 2007). Sendo assim, Dobson \& Dobson (2009), afirmam que no contexto mundial onde os sitemas de saúde buscam e exigem tratamentos eficázes e eficientes na área da saúde mental, a TCC acaba tendo destaque, levando ao desenvolvimento de orientações práticas com forte ênfase no resultados empiricos e limitados pelo tempo, podendo refletir inclusive no melhor aproveitamentoda verba pública.

\section{Terapia Cognitivo-Comportamental na Saúde Pública}

A Terapia Cognitivo-Comportamental no Sistema Único de Saúde pode contribuir de maneira significativa pelo grande números de técnicas que utilizam a breviedade e a objetividade, levando a intervenções mais eficientes e podendo oferecer subsidios para a elaboração de novas políticas públicas em saúde (Mira \& Marques, 2016).

Transtornos mentais como a depressão maior, por exemplo, tem grande impacto na sociedade e na qualidade de vida do paciente, o que sugere a necessidade de uma abordagem 
eficaz na rede de saúde pública. Sendo assim, uma das formas de garantir acesso a intervenções e informações mais eficazes ao usuário, é através da implementação da Terapia Cognitivo Comportamental, que se mostra como um caminho a ser trilhado nos sistemas públicas de saúde (Lopez \& Basco, 2014).

Os sistemas públicos de saúde mental estão indo em direção a tratamentos que são baseados em evidências e que por consequencia refletem de maneira positiva nos gastos públicos. Visto os benefícios da TCC no tratamento do Transtorno Depressivo Maior, a disseminação da abordagem, que é baseada em evidências, necessitaria de uma mobilização e apoio da gestão pública, através do oferecimento de programas de treinamento e supervisão aos psicoterapeutas do setor público (Lopez \& Basco, 2010). Em um estudo realizado por McMartin et al. (2018) em Ontário, a TCC demonstrou também sua eficácia na melhora dos sintomas psicóticos na esquizofrênia em conjunto com anti-psicóticos, efeito esse reportado inclusive por seus familiares. Entretanto os pacientes relataram que o acesso a esse tipo de terapia era limitado.

Outro ponto benefício encontrado no estudo de Eriksson (2017), é a flexibilidade da TCC como ferramenta na atenção básica, onde o uso da terapia de maneira remota, através da internet, surtiu o mesmo efeito do tratamento usual utilizado para depressão nesse ponto de atenção. Medina, Velásquez, Ribeiro \& Trujillo (2018), relatam que a implementação de estratégias e ações preventivas acessíveis e sustentáveis, como a Terapia CognitivoComportamental no combate ao suícido na atenção primária, contribui na redução dos riscos de perda de vida e nos custos financeiros diretos relacionados à intervenção.

Ainda sobre o suicídio, Marback e Pelisoli (2014) afirmam que essa problemática tem sido considerada uma questão de saúde pública no Brasil e no mundo. Os autores ainda relatam que dentre os vários fatores envolvidos no risco do autoextermínio está a desesperença como ponto propulsor, sendo esta um alvo importante na TCC, onde o terapeuta busca gerar algum grau de esperança no paciente, mesmo que seja mínimo, para que o risco seja amenizado.

A psicoeducação, elemento característico na TCC, também pode ser usada como recurso no tratamento em grupo de dependentes químicos (outro grave problema de saúde pública), colaborando no entendimento do paciente sobre sua própria doença e na troca de experiências, contribuindo para a aderencia ao tratamento (Farina, Terroso, Lopes \& Argimon, 2013). Mendes et al.(2016), a partir de uma análise dos custos gerados por um programa de tratamento ao tabagista no Sistema Único de Saúde (SUS) que usufruia da 
abordagem cognitivo-comportamental, constatou um bom desempenho do uso dos recursos públicos no que se refere ao custo-benefício do programa.

Sabe-se que os Sistemas Públicos de Saúde apresentam diversas demandas relacionadas aos seus recursos financeiros limitados, entretanto Revicki et al. (2005) através de uma análise econômica no tratamento para depressão em mulheres de baixa renda no setor público de saúde, constatou que intervenções farmacológicas em conjunto com a Terapia Cognitivo Comportalmental são mais eficazes em termos de custo para o sistema de saúde, além da redução dos sintomas, melhoria no funcionamento e bem-estar dos pacientes.

Além disso, Serfaty et al.(2009) afirma que pessoas idosas com depressão têm grande beneficios com um tratamento individualizado com base na TCC. Demonstrando ser uma abordagem melhor do que a simples conversação com um terapeuta caloroso e empático. Karlin et al. (2018), demonstra que a implementação de um protocolo de TCC para depressão dentro de um sistema de saúde privado refletiu numa redução significativa de sintomas de depressão e ansiedade entre os pacientes.

Especificamente sobre o Sistema Único de Saúde, a TCC pode ser uma forte ferramenta do psicólogo na Saúde Pública, vista sua breviedade e objetividade, podendo respaldar diversas intervenções eficazes para situação em que a rede de saúde mental apresente elevada demanda (Silva, Pereira \& Aquino, 2011).

\section{Considerações Finais}

Pode-se constatar através dos achados na literatura científica, que os sitemas públicos de saúde se beneficiam positivamente com o uso da Terapia Cognitivo-Comportamental, seja nos resultados refletidos da sintomatologia dos pacientes como no controle dos gastos públicos. O resultado positivo no aspecto financeiro pode ser decorrente das características objetivas e adaptativas da abordagem aos diferentes contextos, com limitação de tempo e foco na remissão dos sintomas. Além disso, a TCC apresenta-se como uma aliada da farmacoterapia, onde a psicoeducação entra como fator chave para a aderência ao tratamento, podendo evitar possíveis recaidas por conta do desuso ou possíveis testes que o paciente possa fazer com a medicação.

A padronização de protocolos para a atenção básica baseados em técnicas cognitivocomportamentais apresenta-se como um ponto de inflexão no sistema público de saúde 
brasileiro, sendo extremamento útil num contexto de crise financeira que conta com o agravo da pandemia do novo coronavírus. O custo social e financeiro da reincidência dos transtornos mentais a longo prazo leva a um ciclo vicioso no sistema estatal, que injeta dinheiro em políticas públicas de saúde mental que mantem o paciente preso as amarras da própria patologia.

A flexibilidade dessa abordagem também permite sua aplicação em contextos que a intervenção deva ser feito de maneira remota, via internet por exemplo, sendo útil inclusive no atendimento aos usuários de saúde mental em tempos de pandemia. $\mathrm{O}$ estudo obviamente possuiu limitações, como a presença praticamente nula de estudos sobre a TCC no SUS, sendo que grande parte da literatura obtida através dos descritores foram obtidas de fontes e experiências internacionais. Dessa forma, não há dados suficientes para afirmar como a abordagem se estabeleceria como protocolo para ambulatorios de saúde mental e pontos de atenção básica no SUS.

Por fim, a Terapia Cognitivo-Comportamental não está contra o usuário do sistema público, pelo contrário, está totalmente inclinada a ajudá-lo e a recuperar a sua autônomia, que é obtida também através da remissão parcial ou total dos sintomas, não se limitando apenas a intervenções baseadas no acolhimento do sofrimento, mas na intervenção do mesmo.

\section{Referências}

Bardin, L. (2009). Análise de Conteúdo (4a ed). Lisboa: Edições 70.

Beck, J. S. (2013). Terapia cognitivo-comportamental. Porto Alegre: Artmed Editora.

Brasil (1988). Constituição da República Federativa do Brasil. Brasília: Senado Federal.

Brasil (1990a). Lei $n^{\circ}$ 8.080, de 19 de Setembro de 1990. Dispõe sobre as condições para a promoção, proteção e recuperação da saúde, a organização e o funcionamento dos serviços correspondentes e dá outras providências.

Brasil (1990b). Lei $n^{\circ}$ 8.142, de 28 de Dezembro de 1990. Dispõe sobre a participação da comunidade na gestão do Sistema Único de Saúde (SUS) e sobre as transferências intergovernamentais de recursos financeiros na área da saúde e dá outras providências.

Brasil (2011). Portaria n. 3.088, de 23 de dezembro de 2011. Institui a Rede de Atenção Psicossocial no SUS - RAPS (Republicada em 31 dez. 2011, por ter saído, no DOU n ${ }^{\circ} 247$, 26 dez. 2011, Seção 1, p. 232-233, com incorreção no original). Diário Oficial da União, Brasília-DF, Seção 1, 26 dez. 
Charchat-Fichman, H., Fernandes, C. S., \& Landeira-Fernandez, J. (2012). Psicoterapia neurocognitivo-comportamental: uma interface entre psicologia e neurociência. Revista Brasileira de Terapias Cognitivas, 8(1), 40-46.

David, D., Cristea, I., \& Hofmann, S. G. (2018). Why Cognitive Behavioral Therapy Is the Current Gold Standard of Psychotherapy. Frontiers in Psychiatry, 9. doi:10.3389/fpsyt.2018.00004

Dobson, D., \& Dobson, K. S. (2009). A terapia cognitivo-comportamental baseada em evidências. Porto Alegre: Artmed Editora.

Dobson, K. S.; Dozois, D. J. A. Fundamentos históricos e filosóficos das terapias cognitivocomportamentais. In: Dobson, K. S. et al. (Org.). Manual de terapias cognitivocomportamentais. 2. ed. Porto Alegre: Artmed, 2006, cap. 1, p. 17-39.

Eriksson, M. C., Kivi, M., Hange, D., Petersson, E. L., Ariai, N., Häggblad, P., ... \& Björkelund, C. (2017). Long-term effects of Internet-delivered cognitive behavioral therapy for depression in primary care-the PRIM-NET controlled trial. Scandinavian journal of primary health care, 35(2), 126-136.

Farina, M., Terroso, L. B., Lopes, R. M. F., \& Argimon, I. I. L. (2013). Importância da psicoeducação em grupos de dependentes químicos: relato de experiência. Aletheia, (42), 175185. Recuperado em 05 de julho de 2020, de http://pepsic.bvsalud.org/scielo.php?script=sci_arttext\&pid=S1413-

03942013000300015\&lng=pt\&tlng=pt.

Funcia, F. R. (2019). Subfinanciamento e orçamento federal do SUS: referências preliminares para a alocação adicional de recursos. Ciência \& Saúde Coletiva, 24, 4405-4415.

Jokić-Begić, N. (2010). Cognitive-behavioral therapy and neuroscience: Towards closer integration. Psihologijske teme, 19(2), 235-254.

Karlin, B. E., Brown, G. K., Jager-Hyman, S., Green, K. L., Wong, M., Lee, D. S., ... Ross, T. B. (2018). Dissemination and Implementation of Cognitive Behavioral Therapy for Depression in the Kaiser Permanente Health Care System: Evaluation of Initial Training and Clinical Outcomes. Behavior Therapy. doi:10.1016/j.beth.2018.08.002

Lopez, M. A., \& Basco, M. A. (2014). Effectiveness of Cognitive Behavioral Therapy in Public Mental Health: Comparison to Treatment as Usual for Treatment-Resistant Depression. Administration and Policy in Mental Health and Mental Health Services Research, 42(1), 87-98. doi:10.1007/s10488-014-0546-4

Lopez, M. A., \& Basco, M. R. (2010). Feasibility of Dissemination of Cognitive Behavioral Therapy to Texas Community Mental Health Centers. The Journal of Behavioral Health Services \& Research, 38(1), 91-104. doi:10.1007/s11414-009-9209-8. 
Marback, R. F., \& Pelisoli, C. (2014). Terapia cognitivo-comportamental no manejo da desesperança e pensamentos suicidas. Revista Brasileira de Terapias Cognitivas, 10(2), 122 129. https://dx.doi.org/10.5935/1808-5687.20140018

Mariano, R. B. (2019). O movimento do cientista clínico e a psicologia clínica científica. Revista Brasileira de Terapias Cognitivas, 15(2), 100-111.

McMartin, K., McDowell, S., Gajic-Veljanoski, O., Ali, A., Ekanavake, S., Wells, D. \& Holubowich, C. (2018). Cognitive Behavioural Therapy for Psychosis: A Health Technology Assessment. Ontario health technology assessment series, 18(5), 1-141.

Medina, M., Velásquez, A. V., Ribero, O. J., \& Trujillo, N. (2018). Costos médicos directos por intento de suicidio en pacientes del hospital mental de Antioquia, Colombia. Revista Panamericana de Salud Pública, 42, e129.

Melo, E. A., Almeida, P. F. D., Lima, L. D. D., \& Giovanella, L. (2020). Reflexões sobre as mudanças no modelo de financiamento federal da Atenção Básica à Saúde no Brasil. Saúde em Debate, 43, 137-144.

Mendes, A. C. R., Toscano, C. M., Barcellos, R. M. S., Ribeiro, A. L. P., Ritzel, J. B., Cunha, V. S. \& Duncan, B. B. (2016). Costs of the Smoking Cessation Program in Brazil. Revista de Saúde Pública, 50, 66. Epub November 10, 2016. https://doi.org/10.1590/s15188787.2016050006303

Menezes, A. P. D. R., Moretti, B., \& Reis, A. A. C. D. (2020). O futuro do SUS: impactos das reformas neoliberais na saúde pública-austeridade versus universalidade. Saúde em Debate, 43, 58-70.

Paim, J. S. (2018). Sistema Único de Saúde (SUS) aos 30 anos. Ciência \& Saúde Coletiva, 23(6), 1723-1728.

Pombo, S. \& Ferro, A. (2018). Protocolo cognitivo-comportamental para a perturbação de pânico: eficácia numa amostra portuguesa. Psicologia, Saúde \& Doenças, 19(3), 693709. https://dx.doi.org/10.15309/18psd190318

Rangé, B. P., Falcone, E. M. O, \& Sardinha, A. (2007). História e panorama atual das terapias cognitivas no Brasil. Revista Brasileira de Terapias Cognitivas, 3(2) Recuperado em 05 de julho de 2020, de http://pepsic.bvsalud.org/scielo.php?script=sci_arttext\&pid=S1808$56872007000200006 \& \operatorname{lng}=$ pt\&tlng=pt.

Revicki, D. A., Siddique, J., Frank, L., Chung, J. Y., Green, B. L., Krupnick, J., Miranda, J. (2005). Cost-effectiveness of Evidence-Based Pharmacotherapy or Cognitive Behavior Therapy Compared With Community Referral for Major Depression in Predominantly LowIncome Minority Women. Archives of General Psychiatry, 62(8), 868. doi:10.1001/archpsyc.62.8.868

Rother, E. T. (2007). Revisão sistemática $X$ revisão narrativa. Acta paulista de enfermagem, 20(2), v-vi. 
Santos, R. T. D. (2018a). SUS: um novo capítulo de lutas. Ciência \& Saúde Coletiva, 23, 1719-1720.

Santos, N. R. D. (2018b). SUS 30 anos: o início, a caminhada e o rumo. Ciência \& Saúde Coletiva, 23, 1729-1736.

Serfaty, M. A., Haworth, D., Blanchard, M., Buszewicz, M., Murad, S., \& King, M. (2009). Clinical Effectiveness of Individual Cognitive Behavioral Therapy for Depressed Older People in Primary Care. Archives of General Psychiatry, 66(12), 1332. doi:10.1001/archgenpsychiatry.2009.165

Silva, S. S, Pereira, R. C., \& Aquino, T. A. A. (2011). A Terapia cognitivo-comportamental no ambulatório público: possibilidades e desafios. Revista Brasileira de Terapias Cognitivas, 7(1), 44-49. Recuperado em 05 de julho de 2020, de http://pepsic.bvsalud.org/scielo.php?script=sci_arttext\&pid=S 1808-

$56872011000100008 \& \operatorname{lng}=$ pt\&tlng=pt.

Wright, J. H., Brown, G. K., Thase, M. E., \& Basco, M. R. (2019). Aprendendo a Terapia Cognitivo-Comportamental-: Um Guia Ilustrado. Porto alegre: Artmed Editora.

Wright, J. H., Sudak, D. M., Turkington, D., \& Thase, M. E. (2012). Terapia cognitivocomportamental de alto rendimento para sessões breves: guia ilustrado. Porto Alegre: Artmed Editora.

\section{How to cite this article (APA format):}

Nunes, Rafael Zaneripe de Souza; Tuon, Lisiane; Souza, Rosimeri Vieira da Cruz de; Gomes, Karin Martins (2020). Cognitive-Behavioral Therapy: An Ally of the Unified Health System. Am. In. Mult. $J .$, Jul to Oct. (9) 5, 45-56.

Received: 07/10/2020

Accepted: 07/29/2020 